\title{
A Study on Synthesis Design and Optimization Analysis on Scroll Profiles for Scroll Vacuum Pump
}

\author{
Licun Wang ${ }^{*}, 1,2,3,4$, Xudong Wang ${ }^{1,2}$, Li Du ${ }^{1}$, Xianming Zhang ${ }^{1}$, Bin Chen ${ }^{1}$ and Sinan H. Hussain ${ }^{2,5}$ \\ ${ }^{I}$ Engineering Research Center for Waste Oil Recovery Technology and Equipment of Ministry of Education, Chongqing \\ Technology and Business University, Chongqing, 400067, China \\ ${ }^{2}$ State Key Laboratory of Mechanic Transmission, Chongqing University, Chongqing 400030, China \\ ${ }^{3}$ State Key Laboratory of Tribology, Department of Precision Instruments and Mechanology, Tsinghua University, \\ Beijing 100084, China \\ ${ }^{4}$ National Engineering Research Center of Fluid Machinery and Compressor, Xi'an Jiaotong University, Xi'an 710049, \\ China \\ ${ }^{5}$ Mechanical Engineering Department, Mosul University, Mosul 31313, Iraq
}

\begin{abstract}
The scroll profile plays a very important role in the design of a scroll vacuum pump in oil filter. Therefore, a geometric general form of the scrolls based on Whewell equation was developed in this study. The general planar curve form of the scrolls was specified using the Whewell equation, which consists of three segments and it is easy to analyze and optimize. The analysis reveals that the general model can contain most scrolls based on circle. A general design and multi-objective optimization process for the profile of scroll vacuum pumps was proposed. The design and optimization process was developed based on geometric and multi-objective considerations. The Nondominated Sorting Genetic Algorithm was applied to this multi-objective optimization question. The finite element method/FEM is the kernel of the approach. The scroll components work in a high pressure and high temperature environment, which results in the deformation of the scroll wall. The FEM and optimization can evaluate the deformation due to the cutting force and compensate accordingly.
\end{abstract}

Keywords: Scroll vacuum pump, oil filter, scroll profiles, integration design, optimization analysis.

\section{INTRODUCTION}

With the rapid development of automobile industry and popularity of refrigeration equipment and requirement of many industry such as electron, power, light industry, machinery, chemistry, medical appliance, medical preparation, vintage and food or machining center for compressing medium and other special requirement and the effect of energy shortage, compression theory based on the work principle of conjugation curve engagement and cavity volume changing has been widely studied all over the world $[1,2]$.

Vacuum pump is the key component of oil filter and airconditioning equipment, and the work efficiency of vacuum pump is very important for the whole refrigeration cycle system. The vacuum pump has a great significance in decreasing energy consumption and improving human life quality because of its high efficiency and low noise.

The biggest one of the main problems encountered in the development of a scroll vacuum pump was the design of the

\footnotetext{
*Address correspondence to this author at the College of Mechanical Engineering, No. 19, Xuefu Ave., Nan'an Ditrict, Chongqing Technology and Business University, Chongqing 400067, China; Tel: +86-13983836979 (Mobile), +86-23-62768469(office); Fax: +86-23-62769374; E-mail: wanglicun@yahoo.com.cn
}

scroll profile, which plays a key role in determining the performance of scroll vacuum pumps. To achieve one or some of these above goals, research projects have been conducted during the past twenty years [2,3]. Based on geometric or optimum considerations, many shapes for a scroll compressor have been proposed and demonstrated in papers [4-7]. But few of them consider it on the point of the view of the general form of the scroll and multi-objective optimization. One problem with the traditional design is that compression takes place rather slowly, so a large number of turns is required to achieve the high compression demanded for refrigeration and freezing. The purpose of the study is to found a proper mathematic model for the scrolls, and obtain the optimum scrolls of small size and high compression [815].

The working principle of a scroll vacuum pump is formed by circle spiral. A scroll vacuum pump consists of two spiral scroll elements, which are identical spirals that are assembled at $180^{\circ}$ phase difference [16-22]. The working principle of scroll vacuum pump is illustrated in Fig. (1). They touch at several points and form a series of crescentshaped pockets. One element is stationary and the other rotates in an orbiting plane circular motion around the motor shaft center. The suction fluid is thus brought in simultaneously at two locations from the periphery of the scrolls and is moved continuously inward by the orbiting motion. The volume occupied by the fluid becomes progressively 
smaller and the fluid is steadily compressed. Finally, the fluid is smoothly discharged from a discharge port located near the center of the fixed scroll.
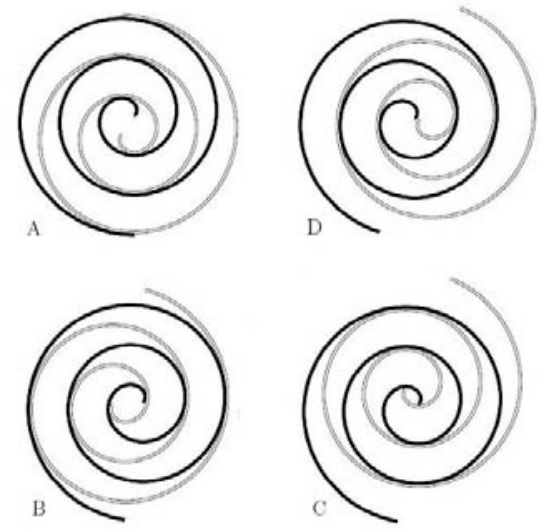

Fig. (1). Working principle of scroll vacuum pump.

In this study, a geometric general form of the scrolls based on Whewell equation is developed. The analysis reveals the general model can contain most scrolls based on circle. A general model of multi-objective optimization is established. The result can meet the demands of better multiperformance.

\section{INTEGRATE DESIGN THEORY OF SCROLL PROFIELS}

The geometry of scrolls is one of fundamental factors influencing the efficiency of the vacuum pump. There are many kinds of curves which meet the conjugate law for planar orbiting mechanisms, such as Archimedean spiral, algebraic spiral, logarithmic spiral, circle spiral, spiral of line segment and square et al. The performance of scroll vacuum pumps is different for different scrolls. For every special scroll, there is an analytic model. In order to analysis easily and achieve the expression for volumes, it is necessary to find a uniform model of scrolls.

From a differential geometric point of view, the inherent characteristics of plane curve lie in the fact that they are completely determined by the intrinsic equation. Furthermore, it is determined uniquely up to a curvature and torsion, and is independent of any choice of coordinates.

The Whewell equation of a general model of scroll can be expressed by a polynomial. A piecewise polynomial or a finite trigonometric polynomial is in $\varphi$. Therefore, a plane curve is represented as shown in Fig. (2). can be described as following formula 1 and 2.

$s(\varphi)=\int_{0}^{\varphi} \rho(u) d u$

$\mathbf{x}(\varphi)=\int_{0}^{\varphi}(\rho(u) \cos (u), \rho(u) \sin (u)) d u$

The above form contains most conjugate curves. If $\rho$ is a polynomial, a piecewise polynomial or just a finite trigonometric polynomial in $\varphi$, and that the parameterization is by tangent direction, both the arc-length $\mathrm{s}$ and the parameterization $x$ can be determined in closed form. Here the spirals of line segment and square are not considered for they are nonregular curves.

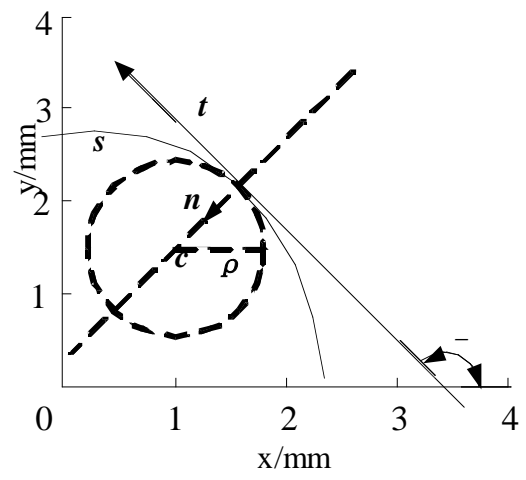

Fig. (2). The definition of geometric variables.

Then, the general geometric form of scrolls is found to be:

$$
\begin{aligned}
& s(\varphi)=\sum_{i=0}^{j} c_{i}\left(\varphi+a_{i}\right)^{i}+p_{i} \cos \left(\varphi+u_{i}\right)+q_{i} \sin \left(\varphi+v_{i}\right) \\
& \left.j \in N, a_{i}, c_{i}, p_{i}, q_{i}, u_{i}, v_{i} \in R\right)
\end{aligned}
$$

From above equation, we can easily extend it to new scrolls. Another advantage is that the optimization model can be established easily, as will be seen later.

Take the commonest scroll of the spiral of circle indicated in Fig. (3) as an example, it can be easily derived from formula 3 by taken $\mathrm{ci}=\mathrm{pi}=\mathrm{qi}=0($ for $\mathrm{i} \neq 2)$, then

$$
p_{i} \cos \left(\varphi+u_{i}\right)+q_{i} \sin \left(\varphi+v_{i}\right)=0
$$

And gained

$$
s(\varphi)=c_{0}\left(\varphi+a_{0}\right)^{2}
$$

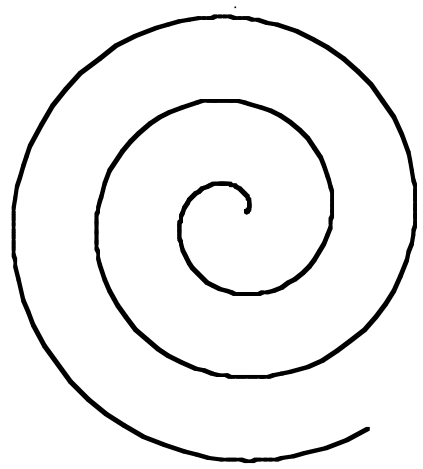

Fig. (3). The standard circle spiral.

After the scroll of fixed wrap is determined, an envelope can be calculated by translating the fixed scroll in a circular orbit with radius $r$. This curve is conjugated with the fixed scroll, so it can be as an orbiting scroll. In consideration that the height and the thickness of the scroll wrap in most vacuum pumps are identical, the volume of the compression chamber is defined as the area enclosed by the spiral of the orbiting and the stationary scroll between two conjugate points, multiplied by the height $h$ of the scroll. This volume can be calculated by the following relationship:

$V(\varphi)=\left\{\pi \cdot r^{2}+r\left[s_{x}(\varphi+2 \pi)-s_{x}(\varphi)\right]\right\} \cdot h$ 
As can be seen from Fig. (3), a condition of an entire compression chamber is that the spiral angle of a turn satisfies $\varphi \geq 2 \pi$. In the case that the orbiting radius $r$ is constant, the compression chamber is determined only by the form of arc-length, that is, it is controlled by the type of scrolls.

There are two symmetrical scrolls assembled together in the vacuum pump here. These two scrolls are offset $180^{\circ}$ and are in conjugation. When any arbitrary pair of points is in conjugation, the wraps are separated by a constant offset distance between the center of the fixed scroll $O_{1}$ and the orbiting scroll $\mathrm{O}_{2}$, which is equal to the orbiting radius $r$, as shown in Fig. (4) and is determined by:

$r=O_{1} O_{2}=(\tilde{n}(\varphi+2 \pi)-\tilde{n}(\varphi)-2 t) / 2$

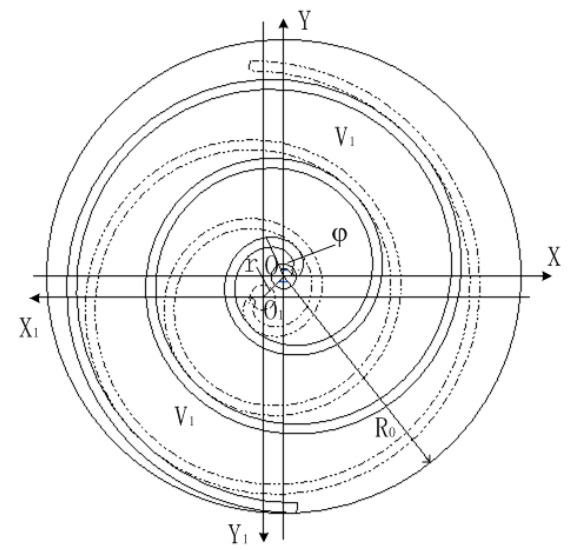

Fig. (4). Two scroll wraps in conjugation.

There are three pairs of compression chamber in a scroll vacuum pump here. The stroke volume ratio is then:

$\gamma=\frac{V(6 \pi)}{V(2 \pi)}$

An important quantity is introduced here, which gives evidence of the usage of scroll bounded area. The normalized stroke volume is defined as:

$$
V_{\text {norm }}=\frac{V(6 \pi)}{\pi \cdot R_{o}^{2}}
$$

\section{OPTIMIZATION ANALYSIS OF SCROLL PRO- FILES}

There are many factors influencing the performance of scroll vacuum pumps. However, when a scroll vacuum pump is used in a limited space such as cars, the stroke volume ratio and the normalized stroke volume become prominent factors. Therefore, the purpose is to design or construct a scroll, which produces a scroll vacuum pump of small size and high efficiency, and this deal with a multi-objective optimization problem. By the using of the general geometric form of scrolls, we can convert a shape optimization into a parameter optimization, and then optimize the coefficients under some constrains, which control the shape of scrolls.

According to above concept, a scroll is constructed following this: at first, from equation 3 , the inner turn $(0$, $m \pi)(2 \leq m<4)$ consists of the standard spiral of circle, and the same to the outer turn $(n \pi, 6.1 \pi)(m<n \leq 4)$, therefore to insure the large compressing ratio. Then the middle turn can be one of arc, algebraic spiral, or other curves, so as to decrease the length of leakage. At the connecting point $(\varphi=m \pi, n \pi)$, the scroll should satisfies continuity. The arc function of scrolls can be described by:

$$
s_{x}(\varphi)=\left\{\begin{array}{rc}
c_{1} \cdot \varphi^{2} & {[0, m \pi]} \\
f\left(c_{1}, c_{2}, \varphi\right) & {[m \pi, n \pi]} \\
c_{2} \cdot \varphi^{2} & {[n \pi, 6.1 \pi]}
\end{array}\right.
$$

Here, assume the biggest angle is $6.1 \pi$.

In which, $f(c 1, c 2, \varphi)$ is one of arc, algebraic spiral, or other curves.

The choice of the function $\mathrm{f}$ of middle turn will determine the scroll shape of middle turn, but can not influence the performance of a scroll vacuum pump, and only influence the construction of second vacuum pump chamber, as can be indicated in Fig. (5).

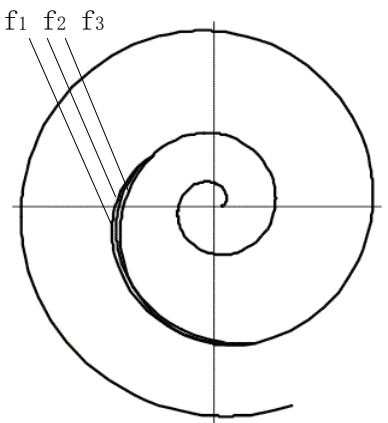

Fig. (5). Influence of middle function on scroll profile.

The value of connecting points $m(2 \leq \mathrm{m}<4), n(\mathrm{~m}<\mathrm{n}<4)$ also can not influence the performance of scrolls. They determine the start and end point of the middle turn, so they influence the volume of second chamber and the rapidness of the compression process. It can be assumed the middle turn is consisted of an arc.

By denoting the design variables $X=[r, c 1, c 2] T$, the two objective optimization functions can be expressed as:

$$
\begin{aligned}
& \max F(X)=\left[F_{1}(X), F_{2}(X)\right]^{T} \\
& \text { s.t. } \quad g_{j}(X) \leq 0 \quad(j=1, \cdots, k)
\end{aligned}
$$

In which, function $\mathrm{F} 1(\mathrm{X})$ is the stroke volume ratio of a scroll vacuum pump and given by:

$$
F_{1}(X)=\gamma(X)=\frac{V(6 \pi)}{V(2 \pi)}
$$

Function $\mathrm{F} 2(\mathrm{X})$ is the normalized stroke volume and described by:

$$
F_{2}(X)=V_{\text {norm }}=\frac{V(6 \pi)}{\pi \cdot R_{o}^{2}}
$$

Function $\mathrm{gj}(\mathrm{X})$ is associated with a number of inequality constrains in the considerations of conjugation and continuity and the satisfaction of equation (5). These conditions can be expanded as follows after the derivation: 
$g_{1}(X)=\pi \cdot c_{1}-0.5 \cdot r-1 \geq 0$

$g_{2}(X)=1-0.5 \cdot \pi \cdot c_{1}+0.25 \cdot r \geq 0$

$g_{3}(X)=r-r_{\min } \geq 0$

$g_{4}(X)=r_{\max }-r \geq 0$

$g_{5}(X)=c_{i}-c_{i \min } \geq 0$

$g_{6}(X)=c_{i \max }-c_{i} \geq 0$

The Nondominated Sorting Genetic Algorithm is applied to this multi-objective optimization question. The GA parameters used in this question are as follows:

Maximum generation: 500

Population size: 100

String length (binary code): 32

Probability of crossover: 0.7

Probability of mutation: 0.001

The calculation results of global Pareto nondominated sets show good convergence. Some of them are listed in Table 1.

Since the performance of above Pareto optimum sets is equal, we can select arbitrarily one optimum plan to analyze. Now the case of the third group in Table $\mathbf{1}$ is selected, so the inner and outer turn of the scroll can be determined. Since the middle turn of the scroll consists of the arc, the distance between the inner and outer wrap of middle turn satisfies the following relationship:

$R_{1}-R_{2}=r$

The wrap thickness of inner turn is given by:

$t_{1}=\pi \cdot c_{1}-r$
And the wrap thickness of outer turn is given by:

$t_{2}=\pi \cdot c_{2}-r>t_{1}$

The scroll of wrap is found to be:

$$
s_{1}(\varphi)=\left\{\begin{array}{lc}
1.7 \varphi^{2} & {[0,3.75 \pi]} \\
(14.5 \cdot \cos (\varphi+1)+48.8) \cdot \varphi & {[3.75 \pi, 4 \pi]} \\
2.45 \varphi^{2} & {[4 \pi, 6.1 \pi]}
\end{array}\right.
$$

Here, assumed $m=3.75 \pi, n=4 \pi$.

We observe the traditional scroll $s 2(\varphi)$ which has the same form as the outer turn of $\operatorname{si}(\varphi)$ :

$s_{2}(\varphi)=2.45 \cdot \varphi^{2} \quad[0,6.1 \pi]$

And $s 3(\varphi)$ which is optimized for $s(\varphi)=c_{3} \varphi 2$ :

$$
s_{3}(\varphi)=1.55 \cdot \varphi^{2} \quad[0,6.1 \pi]
$$

The comparison of geometric properties of the three scroll vacuum pumps are summarized in Table $\mathbf{2}$ and the scrolls are plotted in Fig. (6) as the following.

\section{SOLID MODELING OF SCROLL DISC}

The solid modeling has emerged as a perfect tool for component design. It is more important that solid model can be used as a powerful tool to bridge the gap between the designer and the manufacture engineers, the Pro/Part mode, which is convenient and powerful, is used for the creation of the solid model, to create a vacuum pump solid modeling. The solid modeling is created in the UG/Part environment. In the UG environment the sweep method was used to generate

Table 1. The Numerical Results of Optimization

\begin{tabular}{|c|c|c|c|c|c|}
\hline Rank & Orbiting Radius $\boldsymbol{r} / \mathbf{m m}$ & Coefficient $\boldsymbol{c}_{\boldsymbol{1}}$ & Coefficient $\boldsymbol{c}_{\boldsymbol{2}}$ & The Stroke Volume Ratio $\boldsymbol{\gamma}$ & The Normalized Stroke Volume $\boldsymbol{V}_{\text {norm }}$ \\
\hline \hline 1 & 8.668 & 1.695 & 2.461 & 5.445 & 0.166 \\
\hline 1 & 8.670 & 1.695 & 2.461 & 5.445 & 0.166 \\
\hline 1 & 8.674 & 1.695 & 2.441 & 5.442 & 0.168 \\
\hline 1 & 8.764 & 1.695 & 2.482 & 5.442 & 0.166 \\
\hline 1 & 8.674 & 1.695 & 2.461 & 5.442 & 0.166 \\
\hline 1 & 8.674 & 1.695 & 2.461 & 5.445 & 0.166 \\
\hline 1 & 8.673 & 1.695 & 2.461 & 5.445 & 0.166 \\
\hline
\end{tabular}

Table 2. The Comparison of Performance

\begin{tabular}{|c|c|c|c|}
\hline The Scrolls & Orbiting Radius $\boldsymbol{r} / \mathbf{m m}$ & The Stroke Volume Ratio $\boldsymbol{T}$ & The Normalized Stroke Volume $\boldsymbol{V}_{\text {norm }}$ \\
\hline \hline$s_{1}(\varphi)$ & 8.674 & 5.413 & 0.168 \\
\hline$s_{2}(\varphi)$ & 8.674 & 4.135 & 0.166 \\
\hline$s_{3}(\varphi)$ & 3.350 & 4.210 & 0.132 \\
\hline$\Delta_{1}$ & - & $30.91 \%$ & $1.20 \%$ \\
\hline$\Delta_{2}$ & - & $28.57 \%$ & $27.27 \%$ \\
\hline
\end{tabular}




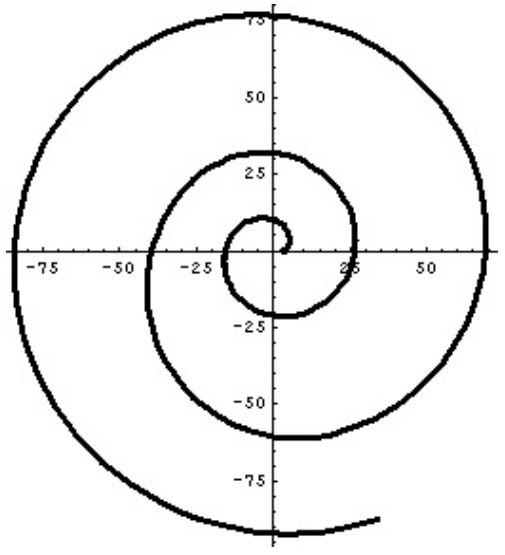

(a) $s_{1}(\varphi)$

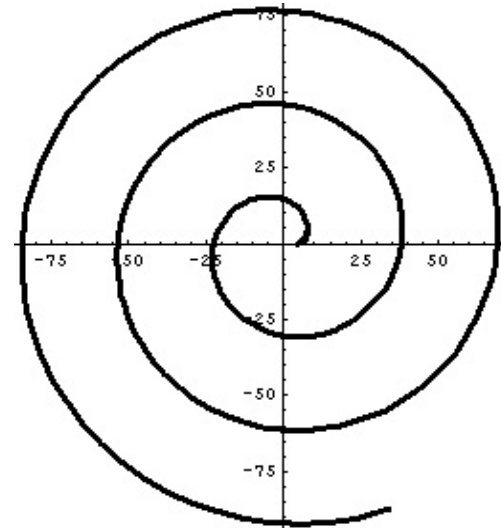

(b) $\mathrm{s}_{2}(\varphi)$

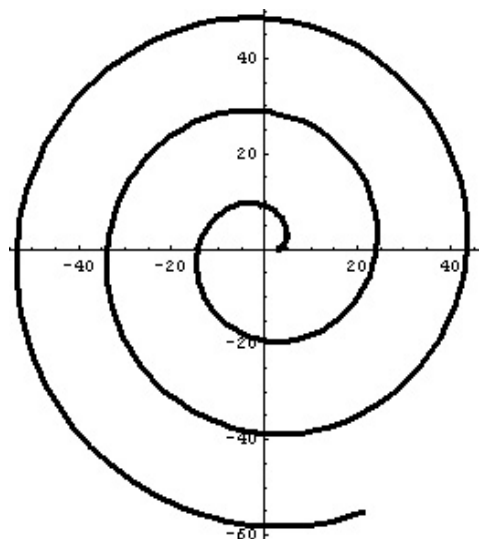

(c) $\mathrm{s}_{3}(\varphi)$

Fig. (6). Three scrolls.

the scroll features as follows. Create the datum plane and coordinate as a base for the features. Select Part-FeatureCreate-Datum-Plane-Default. Then select Create-DatumCoordinate sys-Default. The method to create the general curve is from an equation option by using the above equations or a series of dot data of the general scroll profiles which could be acquired by MATLAB program. In this case the SM of the general scroll profiles can be acquired by the UG by using form file option.

As to optimized profiles of general scroll profiles, according to the theory of control equation of general scroll profiles, the equation of inside and outside profile of orbiting disc and fixed disc are as follows. Now we use the backmost for its better characteristics. In this case we can study the performance, such as vacuum pump capacity, condensing temperature and so on. It is also assumed that the vacuum pump has no discharge valve. The performance of the vacuum pump is determined by the customer's requirements and the design data is gathered from market information. Based on the actual structure of scroll vacuum pump, all the other parts are established through UG, such as Orbiting Disc, Fixed Disc, Vacuum pump Housing, Cross ring, Principal shaft as is shown in the following Fig. (7).

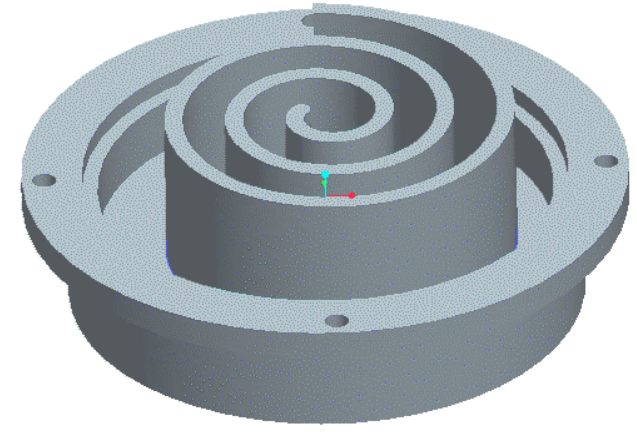

Fig. (7). Stress curve of scroll disc.

\section{CONCURRENT ENGINEERING AND MANUFAC- TURING OF SCROLLS}

The scroll disc is the key part of a scroll vacuum pump. Its deformation will directly affect the performance and reliability of a scroll vacuum pump. Because the structure of orbiting disc is quite complex and the force of various parts is different. However, simple mechanical methods can not be used to calculate the stress and strain, so that finite element analysis techniques should be adopted to analyze and describe the stress and strain of an actual operation of scroll vacuum pumps [5].

The orbiting disc model which is built with UG can be transmitted into ANSYS. The orbiting disc is made of silicon-aluminum alloy with a Young's modulus of $\mathrm{E}=$ $0.073 * 105$ and Poisson's ratio of 0.41 . The number of circle of scroll profile is 2.61 , the height of the scroll wall is 31 $\mathrm{mm}$, the speed of rotation is $1700 \mathrm{r} / \mathrm{m}$, the accelerated velocity is $45 \mathrm{~m} / \mathrm{s} 2$, the pressure of inspiration is $0.113 \mathrm{MPa}$, and pressure of air discharge is $2.216 \mathrm{MPa}$. In order to accurately reflect the displacement field and stress field of disc as shown in Fig. (8), the best way is to set all structure of the disc, including subtle chamfering and others as study subjects to study the important structure. The manufacturing process and the type model are shown in Fig. (9).

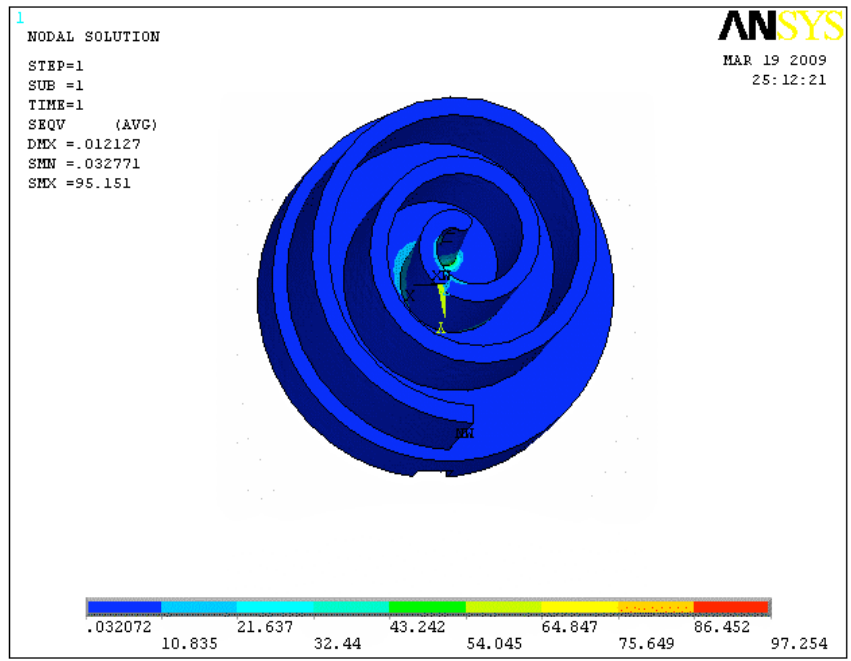

Fig. (8). Scroll disc of vacuum pump.

It can operate on different speed of rotation or deferent gas discharge pressure. The static analysis for the structures 

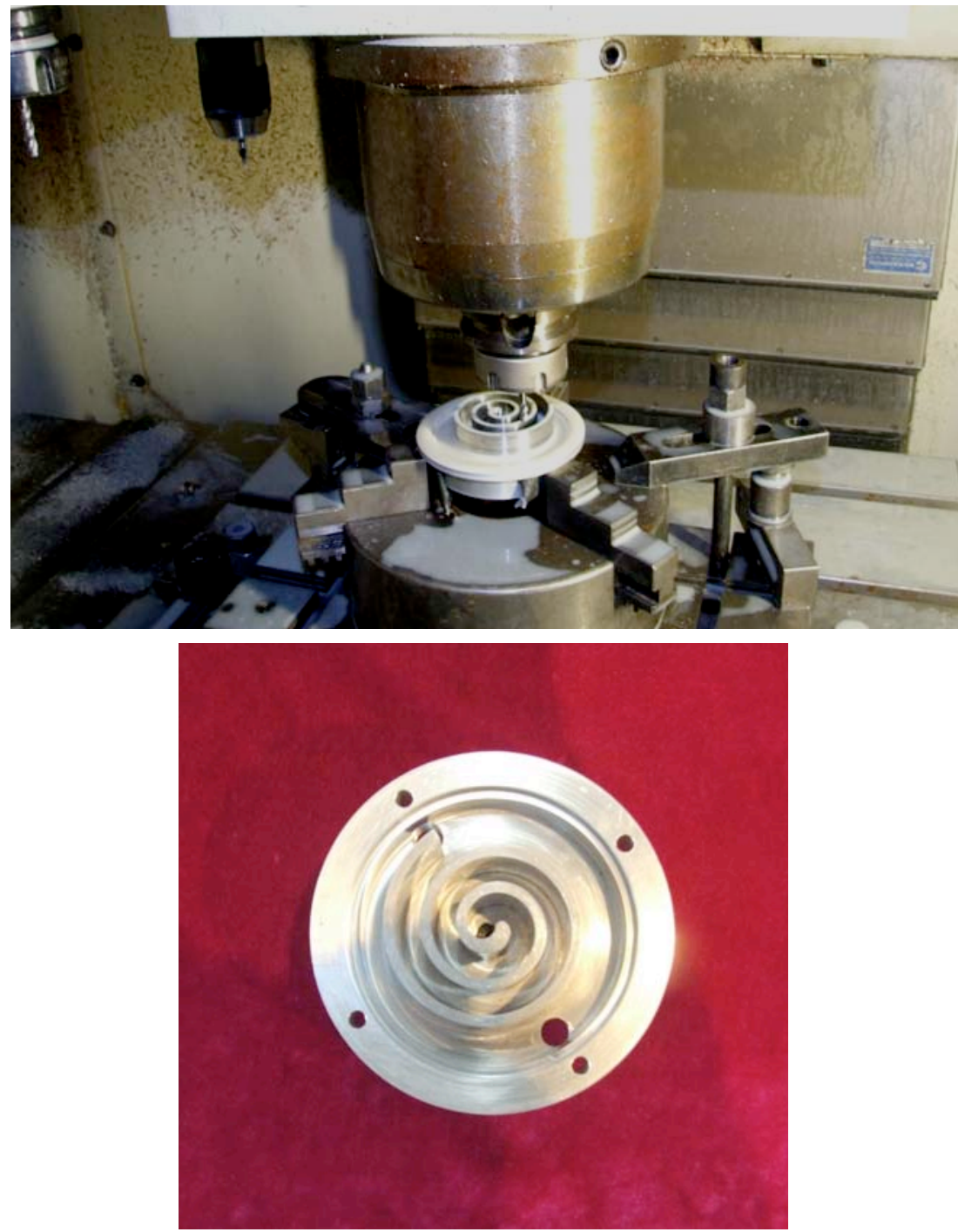

Fig. (9). Manufacturing process and the scroll model.

of scroll vacuum pumps is adopted for simplification through finite element method, because it is simpler than dynamic analysis. And one or several working conditions should be chosen to calculate. That structures which can bear the greatest pressure, the biggest pulling force, the largest blending or maximum torque and so on are chosen as commonly used calculation working conditions. The deformation of scroll tooth is very small. In this thesis, the three-dimensional four nodes unit is analyzed. Scroll vacuum pumps can generally operate on different conditions. Compared with scroll tooth, the scroll end-plate is thicker, and its surrounding structure plays a role of strengthening, so its actual deformation is very small, it can be considered as a rigid body so as to be calculated easily.

The orbiting disc not only subjects two kinds of force load, but also subjects to temperate load. The gas temperature in the outside scroll cavity is lower, and the gas in the central scroll cavity is higher. Weather the temperate is lower or higher is related to the degree of compression of gas, therefore, the temperate rises from outside to centre. During the working process of a scroll vacuum pump, the load of orbiting disc mainly contains two parts: the temperature load, the inertial load, kinds of gas load, and contact load. The orbiting disc while operating mainly bears gas axial force, radial force, and tangential force, contact force between orbiting disc and fixed disc and gravity force. During working process, pressure in the outside suction housing of orbiting disc is suction pressure. Pressure in the central cavity which connected to the gas discharge outlet is discharge pressure. On scroll tooth, the radial force only exist on that part where there are pressure difference between outside and inside, gas axial force exist on the end-plate of orbiting disc, and it gradually change from the outside suction pressure to central discharge pressure.

Displacement increases gradually along the scroll wall from the outside to the inside and from the bottom to the top. The reason is that the pressure in discharging chamber is higher than the pressure in the suction chamber. This displacement result is very important for the scroll assembly clearances. Because the orbiting scroll subjects to both axial gas force and radial gas force, as a result, orbiting scroll will produce synthetic bending transformation and the transformation at the head of tooth nearby is the maximum of $12 \mu \mathrm{m}$. Uniting displacement transformation of orbiting scroll is within promised range. 


\section{CONCLUTIONS}

In the study on the profile design and optimization of scrolls, a general form based on the Whewell equation is proposed, and a detailed geometric design model and optimization process is developed. The Nondominated Sorting Genetic Algorithm is applied to this multi-objective optimization question. A scroll that can meet the demand of better multi-performance is obtained. The study is based on the consideration of geometry and optimization. The influence of dynamics and thermodynamics was not probed, and might be considered in the future to refine the model further.

Concurrent engineering combined with information technology is used for the scroll vacuum pump computer-aided design and UG software is used for the main role in the approach in the research of scroll profiles for scroll vacuum pump. The finite element method was carried out in ANSYS and the transformation at the head of tooth nearby is the maximum of $11 \mu \mathrm{m}$ within promised range 86Mpa.

The method is not only fit for the scroll profiles statement above, but also fit for studying other correlation profiles of scrolls. It offers mathematic model for studying performance characteristics and efficiency of other scroll profiles. It is a very important theory and is of practical significance and the research idea develops the method to the profile design of scroll profiles for vacuum pump.

\section{ACKNOWLEDGEMENTS}

The financial support of the National Natural Science Foundation of China (50805149), China Postdoctoral Science Foundation (20090450166, 201003669), State Key Lab of Mechanical Transmission Open Foundation of China, Chongqing Municipal Intellectual Property Office Special Foundation, Chongqing Science and Technology Commission Scientific and Technological Project Foundation (CSTC2009AB3234/CSTC2011GGC246) and Chongqing University Innovation Group Team Project (KJTD201019) are gratefully acknowledged.

\section{REFERENCES}

[1] Y. Cui, Y. Arai, T. Asai, B. Ju, and W. Gao, "A high-speed atomic force microscope for precision measurement of microstructured surfaces", International Journal of Precision Engineering and Manufacturing, vol. 9, pp. 27-32, March 2008.

[2] W. Gao, Y. Arai, A. Shibuya, S. Kiyono, and C. H. Park, "Measurement of multi-degree of freedom error motions of a precision linear air-bearing stage", Precision Engineering, vol. 30, pp. 96-103, January 2006.

[3] Y. Chen, N. Halm, and E. Groll, "Mathematical modeling of scroll compressors part I: compression process modeling", International Journal of Refrigeration, vol. 25, pp. 731-750, July 2002.

[4] W. Gao, S. Dejima, Y. Shimizu, and S. Kiyono, "Precision measurement of two-axis positions and tilt motions using a surface encoder", Annals of the CIRP, vol. 52, pp. 435-438, January 2003.
W. Gao, T. Araki, S. Kiyono, Y. Okazaki, and M. Yamanaka, "Precision Nano-fabrication and evaluation of a large area sinusoidal grid surface for a surface encoder", Precision Engineering, vol. 27, pp. 289-298, March 2003.

[6] Q. S. Lee, and G. D. Gweon, "A new 3-DOF Z-tilts micro positioning system using electromagnetic actuators and air bearings", Precision Engineering, vol. 24, pp. 24-31, January 2000.

[7] J. Chen, L. C. Wang, and S. L. Li, "Study and profound analysis on general profile theory of scrolls", Jixie Gongcheng Xuebao/Chinese Journal of Mechanical Engineering, vol. 42, pp. 11-15, May 2006.

[8] W. Gao, D. Shuichi, Y. Hiroaki, K. Kei, K. Satoshi, and T. Yoshiyuki, "A surface motor-driven planar motion stage integrated with an XYZ surface encoder for precision positioning", Precision Engineering, vol. 28, pp. 329-337, March 2004.

[9] Y. Watanabe, W. Gao, H. Shimizu, and S. Kiyono, "Analysis of a surface encoder in wave optics", Key Engineering Materials, vol. 257-258, pp. 219-224, May 2004.

[10] W. Gao, S. Dejima, and S. Kiyono, "A dual-mode surface encoder for position measurement”, Sensors and Actuators A, vol. 117, pp. 95-102, January 2005.

[11] L. C. Wang, J. Chen, S. L. Li, and J. X. He. "Conjugate meshing theory of scroll profiles based on functional expression", Jixie Gongcheng Xuebao/Chinese Journal of Mechanical Engineering, vol. 43, pp. 50-53, March 2007.

[12] H. Qiu, A. Kubo, Z. Li, and H. Ikegami, "Measurement of machining center motion trajectories within a small area", Transactions of the Japan Society of Mechanical Engineers, vol. 716, pp. 1034-1040, July 2006.

[13] H. Chiu, H. Ozaki, E. Sato, T. Suzuki, A. Oho, and Y. Ariura, "An analysis using offset curves for profiles manufacturing and errors of plane cams. JSME International Journal, vol. 36C, pp. 110-118, January 1993.

[14] H. Qiu, Y. Li, and Y. B. Li, "A new method and device for motion accuracy measurement of NC machine tools. Part 1: principle and equipment", International Journal of Machine Tools and Manufacture, vol. 41, pp.521-534, April 2001.

[15] H. Qiu, Y. Li, and Y. B. Li, "A new method and device for motion accuracy measurement of NC machine tools. Part 1: principle and equipment", International Journal of Machine Tools and Manufacture, vol. 41, pp. 535-554, April 2001.

[16] Z. Jiang, K. Cheng, and D. K. Harrison, "A concurrent engineering approach to the development of a scroll compressor", Journal of Materials Processing Technology, vol. 107, pp. 194-200, January 2000

[17] Z. Jiang, D. K. Harrison, and K. Cheng, "Computer-aided design and manufacturing of scroll compressors", Journal of Materials Processing Technology, vol. 138, pp. 145-151, January 2003.

[18] K. C. Fan, and M. J. Chen, "A 6-degree-of-freedom measurement system for the accuracy of X-Y stages”, Precision Engineering, vol. 24, pp. 15-23, January 2000.

[19] Q. S. Lee, and G. D. Gweon, "A new 3-DOF Z-tilts micro positioning system using electromagnetic actuators and air bearings", Precision Engineering, vol. 24, pp. 24-31, January 2000.

[20] C. Yu, "Mathematical modeling of scroll compressors-part 1: compression process modeling", International Journal of Refrigeration, vol. 25, pp. 731-750, June 2002.

[21] L. C. Wang, J. Chen, Y. Y. Zhao, and S. H. Hussain, "Research on the natural meshing mechanism and testing analysis of scroll profiles for refrigeration scroll compressor", Science China, vol. 53, pp. 2783-2791, October 2010.

[22] L. C Wang, J. Chen, S. L. Li, and J. X. He, "Conjugate meshing theory of scroll profiles based on functional expression", Chinese Journal of Mechanical Engineering, vol. 43, pp. 50-53, March 2007. 